\title{
Shifting the Universe: Early Dark Energy and Standard Rulers
}

\author{
Eric V. Linder ${ }^{1}$ and Georg Robbers ${ }^{2}$ \\ ${ }^{1}$ Berkeley Lab \& University of California, Berkeley, CA 94720, USA \\ ${ }^{2}$ Institut für Theoretische Physik, Philosophenweg 16, D-69120 Heidelberg, Germany
}

(Dated: December 4, 2018)

\begin{abstract}
The presence of dark energy at high redshift influences both the cosmic sound horizon and the distance to last scattering of the cosmic microwave background. We demonstrate that through the degeneracy in their ratio, early dark energy can lie hidden in the CMB temperature and polarization spectra, leading to an unrecognized shift in the sound horizon. If the sound horizon is then used as a standard ruler, as in baryon acoustic oscillations, then the derived cosmological parameters can be nontrivially biased. Fitting for the absolute ruler scale (just as supernovae must be fit for the absolute candle magnitude) removes the bias but decreases the leverage of the BAO technique by a factor 2 .
\end{abstract}

\section{INTRODUCTION}

The acceleration of the cosmic expansion occurs in the relatively recent universe, at redshifts $z<1$ when the universe is several billion years old. However, the origin of the dark energy responsible for the acceleration is unknown and we have no guarantee that it is of purely recent origin. Early dark energy models can be attractive both theoretically and observationally, and should not be neglected when interpreting cosmological data lest the conclusions be biased. Here we consider the role that early dark energy (EDE) plays in high redshift observations of the cosmic microwave background (CMB) and baryon acoustic oscillations (BAO), and the use of the baryon-photon fluid sound horizon as a standard ruler.

In fact, for any cosmological probe tied to high redshift one must know the expansion history, i.e. the dark energy properties, over the whole range or even the low redshift conclusions will be biased (see, e.g., [1]). One cannot assume the dark energy "fades away": one must allow for the possibility of its early existence and test for this. Recognition of the impact of early dark energy is crucial for proper implementation of the current CMB shift parameter and BAO data constraints in cosmological parameter fitting, as illustrated in [2, 3] for example. The effects on growth of structure are even more dramatic; see for example [4, 5].

CMB measurements are quite precise and the sound horizon is robust against many cosmological modifications, as shown by [6], but they also identified that the early expansion history represents a loophole in this. Early dark energy contributing a fraction $\Omega_{e}$ to the total energy density shifts the sound horizon by a factor $\left(1-\Omega_{e}\right)^{1 / 2}$ [7]. By itself this would be precisely measured in $\mathrm{CMB}$ data; however the distance to $\mathrm{CMB}$ last scattering changes as well and could compensate such that both shifts went undetected [8].

In this paper we examine the effects on CMB and BAO data more closely. Describing early dark energy in \$I we investigate in $\cong$ III first the geometric degeneracy between the sound horizon and the distance to last scattering and then carry out a Markov Chain Monte Carlo analysis of the $\mathrm{CMB}$ power spectra to give a detailed comparison of early and standard fading dark energy cosmologies. In IV] we examine the consequences of unrecognized shifts in the cosmology for the BAO method employing the biased standard ruler.

\section{EARLY DARK ENERGY MODEL}

Early dark energy has motivations on both the theoretical and observational fronts. Tracking quintessence [9] attempts to alleviate the coincidence problem of the cosmological constant by considering a dynamical scalar field with a potential of the form that brings the field evolution onto an attractor trajectory, greatly enlarging the range of initial conditions that can deliver a present dark energy density near $70 \%$ of the critical energy density. This has the consequence that the energy density is a nonnegligible fraction of the matter density during the matter dominated era, including the recombination epoch. Dilaton fields giving rise to an exponential potential [10], appearing in many particle physics models, have such a form and indeed the dark energy density traces the dominant energy density as a constant fraction.

In addition to persistent, early dark energy some possible sources for deviation in the sound horizon paradigm include a post-nucleosynthesis acceleration as in stochastic dark energy models 11, 12] or nonminimal couplings such as in scalar-tensor [13] or coupled dark energy theories [14]. However we concentrate on EDE as the simplest and directly motivated model.

Constraints from primordial nucleosynthesis and from the CMB limit the early dark energy fraction to be small, less than about 4\% [15], but not completely negligible. Indeed, EDE at the $1 \%$ level can have significant effects on formation of massive structures [4] and may help explain the early onset of star and galaxy formation as well as the high level of Sunyaev-Zel'dovich effect contribution to the high multipoles of the CMB temperature power spectrum [16]. Note that in contrast, a cosmological constant has a fractional energy density at the $10^{-9}$ level at $z \approx 10^{3}$.

To capture the physical effects of dark energy at both 
recent and early epochs we adopt the form [17]

$$
\Omega_{D E}(a)=\frac{\Omega_{D E}-\Omega_{e}\left(1-a^{-3 w_{0}}\right)}{\Omega_{D E}+\Omega_{m} a^{3 w_{0}}}+\Omega_{e}\left(1-a^{-3 w_{0}}\right)
$$

for the dark energy density as a function of scale factor $a=1 /(1+z)$. Here $\Omega_{D E}$ is the present dark energy density, $\Omega_{e}$ is the asymptotic early dark energy density, $w_{0}$ is the present dark energy equation of state, or pressure to energy density ratio, and $\Omega_{m}\left(=1-\Omega_{D E}\right)$ is the present matter density.

\section{CMB POWER SPECTRA}

The sound horizon of the coupled photon-baryon fluid plays a critical role in the physics of density perturbations in both the photons (which translate into the temperature perturbations seen through the CMB temperature power spectrum) and baryons (which appear as baryon acoustic oscillations, seen through the statistical pattern of galaxy positions, today on $100 h^{-1} \mathrm{Mpc}$ scales). The sound horizon $s$ is given by

$$
s=\int_{z_{d e c}}^{\infty} d z \frac{c_{s}}{H(z)}
$$

where $z_{\text {dec }}$ is the redshift of decoupling between the photons and baryons, $c_{s}$ is the sound speed of the fluid, depending on the baryon and photon densities, and $H(z)$ is the Hubble parameter, depending on the energy densities of all components, not just baryons and photons.

As mentioned, in $\Lambda \mathrm{CDM}$ the cosmological constant contributes less than $10^{-9}$ of the dominant term in $H(z)$, and even for a dark energy equation of state $w=-2 / 3$ the contribution is of order $10^{-6}$. The Hubble parameter for a universe with early dark energy in addition to the usual (matter and radiation) components is given by

$$
\begin{aligned}
H^{2}(z) & =\frac{8 \pi}{3}\left[\rho_{\mathrm{noEDE}}(z)+\rho_{\mathrm{EDE}}(z)\right] \\
& =\frac{8 \pi}{3} \rho_{\mathrm{noEDE}}(z)+H^{2}(z) \Omega_{\mathrm{EDE}}(z) \\
& =\frac{8 \pi}{3} \frac{\rho_{\mathrm{noEDE}}(z)}{1-\Omega_{\mathrm{EDE}}(z)} .
\end{aligned}
$$

Thus we see that for EDE scaling as a constant fraction of the energy density that at high redshift $H(z) \sim$ $\left(1-\Omega_{e}\right)^{-1 / 2}$ [18]. Holding $z_{\text {dec }}$ fixed (it depends predominantly on atomic physics and is quite robust to changes in dark energy) and $c_{s}$ fixed (it involves baryonic physics), then we immediately see that

$$
\frac{s_{\mathrm{EDE}}}{s_{\mathrm{noEDE}}} \sim\left(1-\Omega_{e}\right)^{1 / 2} .
$$

Thus, early dark energy shifts the sound horizon.

Furthermore, EDE shifts distances, for example the conformal distance

$$
d(z)=\int_{0}^{z} \frac{d z^{\prime}}{H\left(z^{\prime}\right)}
$$

but the crucial point is that because the dark energy contributes different amounts to $H(z)$ at different redshifts, the shifts are not uniform but vary with redshift. Thus, the measured distance ratio, or angular scale, of the distance relative to the sound horizon scale, is preserved for only one particular distance, depending on the early dark energy density and other cosmological parameters.

For some parameter values, the ratio of distance to CMB last scattering relative to the sound horizon, $d_{\mathrm{lss}} / s$, is conserved, leaving untouched the geometric factor determining the measured location of the CMB acoustic peaks. This has two implications: one cannot distinguish early dark energy from standard fading dark energy in this case from the CMB acoustic scale, and then one unavoidably obtains a shift in the ratio of sound horizon to distances at other redshifts - precisely what enters BAO. Without specifically fitting for the possibility of EDE therefore, the shift goes unrecognized, effectively miscalibrating the distances, and pointing to a spurious cosmology.

To calibrate the standard ruler of the sound horizon for $\mathrm{BAO}$ cosmology, one must therefore either discern signatures of EDE in the CMB even with $d_{\mathrm{lss}} / s$ matched, or employ independent observations, such as the amplitude of growth and nonlinear structure. We concentrate here on the former, since precision measurements of growth are not as advanced.

We now investigate whether one can robustly recognize models with EDE distinctly from fading dark energy cosmologies by examining the CMB power spectra for those models even when the last scattering distance ratio is matched. For example, the CMB peak structure is not a purely periodic oscillation but is driven by the gravitational potentials. Since EDE is essentially unclustered at these early times, it does not participate in the driving as dark matter does, giving an early Sachs-Wolfe effect that alters the rise to the first peak ${ }^{1}$. However, this might in turn be compensated by tilting the scalar index, which then has further physical effects that might be compensated by changing other parameters.

Figure 1 demonstrates just how exactly models with EDE can reproduce the CMB power spectra of a fiducial fading dark energy model. For Fig. 1, we chose the CMB power spectra of a $w_{0}=-0.95, w_{a}=-0.1$ model without EDE as the fiducial spectra, and then used the Boltzmann code cmbeasy [20, 21] to find EDE models with $\Omega_{e}=0.03, w_{0}=-0.95$ that match the fiducial spec$\operatorname{tra}^{2}$. To find matching spectra, we scanned the parameter space via Markov Chain Monte Carlo (MCMC) runs,

\footnotetext{
1 Note that attributing the effect of EDE to the early Sachs-Wolfe effect, as opposed to say the Doppler rise from the perturbation velocities, is a gauge dependent statement. See [19] for a review of the various physical effects that contribute to the CMB power spectrum.

2 We chose $w_{a}=-0.1$ to match the CMB distance ratio of the EDE model with $\Omega_{e}=0.03$ but all other parameters fixed.
} 


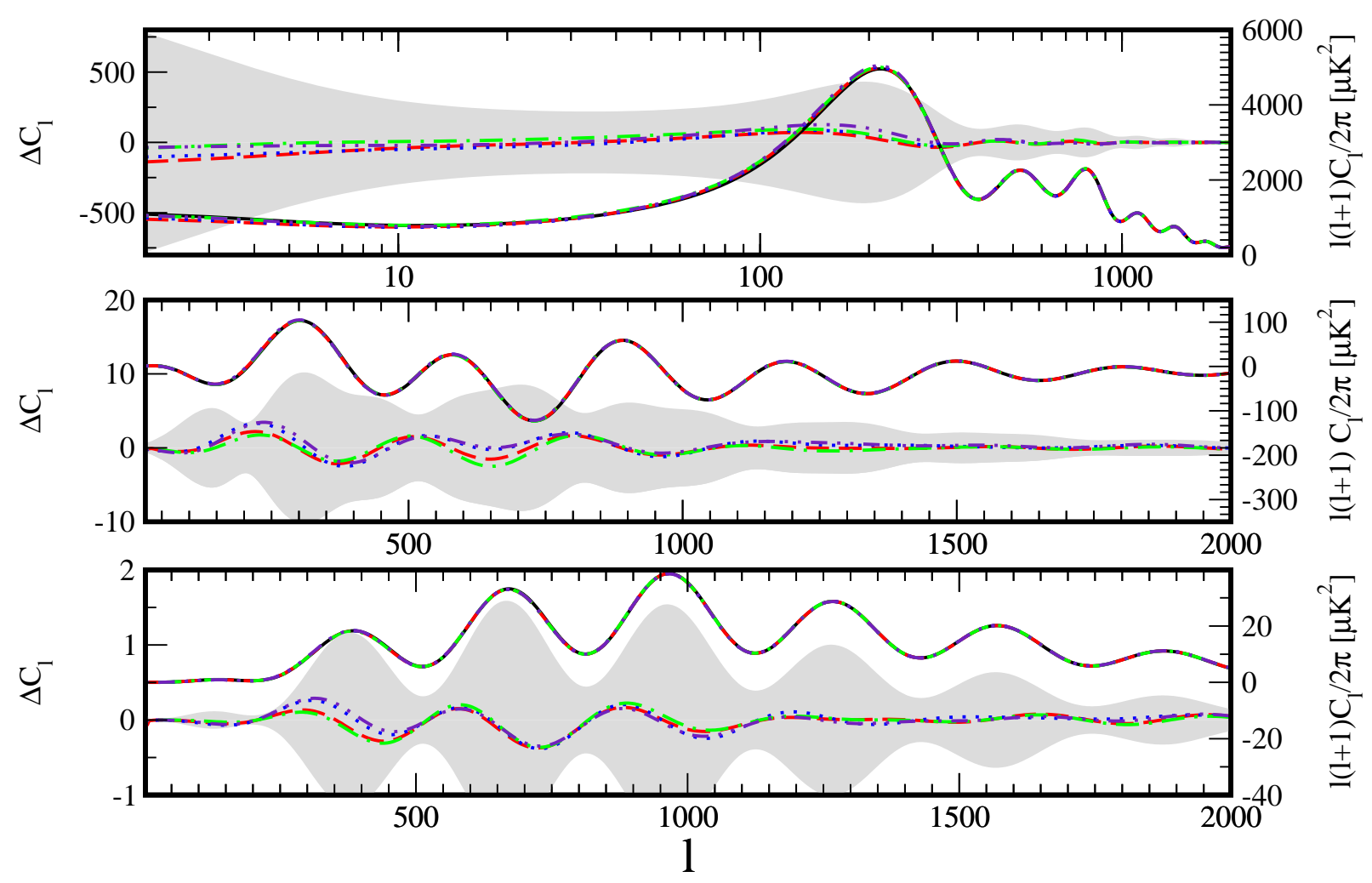

FIG. 1: CMB power spectra for TT (top panel), TE (middle), and EE (bottom) modes of different $\Omega_{e}=0.03$ EDE models (listed in Table I) are plotted using the right axis scale. Their differences relative to the fiducial $\left(w_{0}=-0.95, w_{a}=-0.1\right)$ model are shown using the left axis scale, and lie within the grey shading surrounding the zero difference level that shows the cosmic variance limit.

\begin{tabular}{|c|c|c|}
\hline fiducial & & $\begin{array}{l}w_{0}=-0.95, w_{a}=-0.1 \\
\text { no } \mathrm{EDE}\end{array}$ \\
\hline A & - & $\begin{array}{l}\text { matching } 2<C_{\ell}^{T T}< \\
2000\end{array}$ \\
\hline B & $\bullet \bullet ・$ & $\begin{array}{l}\Omega_{m}=0.28, \text { matching } \\
2<C_{\ell}^{T T}<2000\end{array}$ \\
\hline $\mathrm{C}$ & - & $\begin{array}{l}\text { matching } 2<C_{\ell}^{T T, E E}< \\
2000\end{array}$ \\
\hline $\mathrm{D}$ & $\bar{\square} \cdot \bullet$ & $\begin{array}{l}\Omega_{m}=0.28, \text { matching } \\
2<C_{\ell}^{T T, E E}<2000\end{array}$ \\
\hline
\end{tabular}

TABLE I: Fitting requirements for the models shown in Fig. 1

varying the physical baryon density $\Omega_{b} h^{2}$, physical matter density $\Omega_{m} h^{2}$, Hubble constant $h$, optical depth $\tau$, power spectrum amplitude $A_{s} e^{-2 \tau}$, and the scalar spectral index $n_{s}$ of the EDE models.

The examples shown are from Monte-Carlo chains trying to match the TT-spectra in the range $2<\ell<2000$ (model A), plus making the match more challenging by requiring exact agreement on $\Omega_{m}$ (model B), or matching both TT and EE-spectra (model C), plus exact $\Omega_{m}$ (model D) (also see Table 【). As seen, the CMB power spectra of models containing substantial early dark energy, here $\Omega_{e}=0.03$, can mimic a cosmology lacking early dark energy. That is to say, the shift in the sound horizon and subsequent miscalibration of the standard ruler can be hidden.

For all matching requirements, the comparison precision assumed was for a experiment covering a fraction of $81 \%$ of the sky, limited only by cosmic variance up to $\ell=2000$, which Planck will approach for the TT power spectrum. The overall $\chi^{2}$ for the TT-spectrum of model A relative to the fiducial model, for six parameters over nearly 2000 degrees of freedom, is 14 . Note for comparison that WMAP 5 year data gives a $\chi^{2}$ of $\sim 1000$ relative to their best fit $\Lambda \mathrm{CDM}$ model for unbinned multipoles over the range $\ell=33-1000$ [22]. Their reduced $\chi^{2}$ with binned multipoles is 1.04 ; using the same binning the EDE model has a reduced $\chi^{2}$ of 0.3 . Moreover, we have considered only a limited set of potential degeneracies, for example holding $w_{0}$ fixed and not including running or spatial curvature, so further degeneracies exist that could aid in hiding early dark energy.

Two possible routes to breaking the degeneracies that prevent recognition of EDE are use of the B-mode spectrum, whose lensing component measures growth of structure, affected by EDE as mentioned above (although note that the ISW effect already included also probes growth), and precision measurement of the Hubble constant $H_{0}$, that shifts to partially compensate for the 


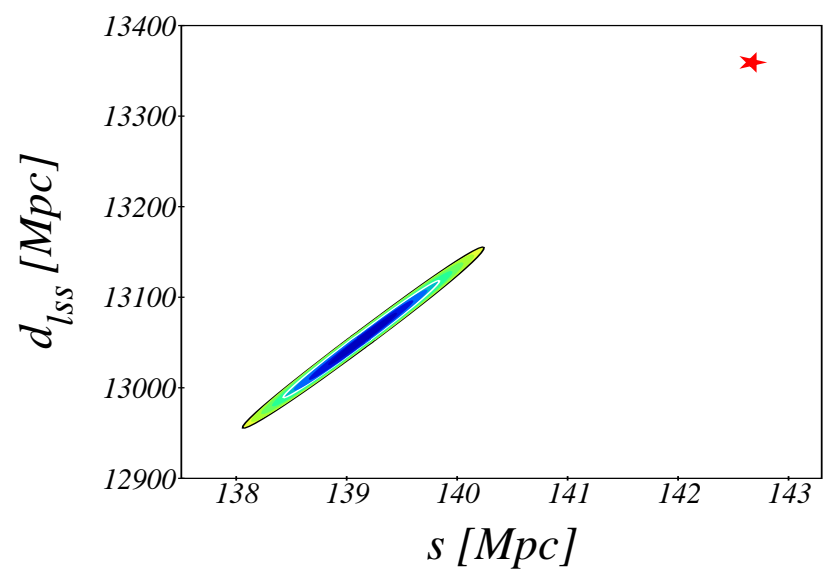

FIG. 2: Likelihood contour at $95.4 \% \mathrm{cl}$ in the $s-d_{\mathrm{lss}}$ plane for EDE cosmologies with $\Omega_{e}=0.03$ that match the CMB temperature power spectrum of the fiducial $\left(w_{0}=-0.95, w_{a}=-0.1\right)$ model without early dark energy. Note that the matching almost automatically preserves the geometric shift factor, i.e. the slope $d_{\mathrm{lss}} / s$, but not the individual distance scales. The red star marks $s$ and $d_{\mathrm{lss}}$ of the fiducial model; the shift in the standard ruler amounts to $2.5 \%$.

sound horizon alteration.

It is also interesting to note the implication that the physical matter density $\Omega_{m} h^{2}$ entering predictions for dark matter direct detection signals may differ from the value from standard CMB constraints. Since the nonrelativistic matter density needed to fit the CMB data gains an additional contribution from EDE, i.e. is $\left(\Omega_{m}+\Omega_{e}\right) h^{2}$ if $\mathrm{EDE}$ had $w=0$ (but in fact $w$ around recombination is often slightly positive), then the quantity $\Omega_{m} h^{2}$ may differ from what the standard analysis might suggest.

The MCMC results presented here demonstrate that the presence of EDE (even with the rather large value of $\Omega_{e}=0.03$ ) may not be cleanly distinguished from standard fading dark energy, even by cosmic variance limited CMB experiments similar to Planck. In the next section we investigate the implications if such a miscalibration were to occur.

\section{NONSTANDARD RULER}

Cosmological probes such as BAO that rely on the sound horizon (or any other early universe quantity) as a standard ruler will be miscalibrated in the presence of unrecognized EDE. Both the sound horizon $s$ and distances $d$ to various redshifts are altered. It is the ratio of these that provides cosmological tests. Figure 2 illustrates the dramatic shifts in each quantity; note that the shift exceeds that in Eq. (6) because other parameters change between the fit and fiducial models. The figure also demonstrates how the shifts can be hidden through preservation of the CMB acoustic peak scale given by the ratio of the distance to last scattering to the sound horizon scale, $d_{\mathrm{lss}} / s$.
The effect of the miscalibration of the sound horizon standard ruler in the distance ratio varies with redshift and so can mimic changes in the cosmology. We examine the propagation of this miscalibration in $\mathrm{BAO}$ analysis into the biasing of cosmological parameter estimation. In particular, we consider the effect on the derived dark energy equation of state parameters of the form

$$
w(z)=w_{0}+w_{a}(1-a) .
$$

Within the Fisher information formalism, a miscalibration in the measured quantities $O_{k}$ propagate into the cosmological parameters $p_{i}$ as

$$
\delta p_{i}=\left(F^{-1}\right)_{i j} \sum_{k} \Delta O_{k} \frac{\partial O_{k}}{\partial p_{j}} \frac{1}{\sigma^{2}\left(O_{k}\right)},
$$

approximating measurement errors in the observables as uncorrelated. For BAO the "observables" are taken to be the ratio of the angular diameter distance at some redshift $z_{k}$ to the sound horizon, $\tilde{d}=d / s$, and the ratio of the proper distance interval (essentially $H^{-1}\left(z_{k}\right)$ for infinitesimal intervals) to the sound horizon, $\tilde{H}^{-1}=H^{-1} / s$. These arise from measurement of the transverse and radial BAO modes, respectively.

Early dark energy shifts the sound horizon, the distances, and the distance intervals, and from these generates the offsets $\Delta O_{k}$. The bias on the derived cosmology due to the miscalibration is illustrated in Fig. 3, Here we take a true cosmology with $\Omega_{e}=0.03, \Omega_{m}=0.28$, $w_{0}=-0.95$ and consider the results of a $\mathrm{BAO}$ experiment achieving $1 \%$ precision on both $\tilde{d}$ and $\tilde{H}^{-1}$ at redshifts $z=0.4,0.6,0.8,1$. Note that this is quite optimistic, being beyond the statistical power of the proposed BOSS and WFMOS surveys even without accounting for systematics. We also include CMB measurements of the quality of Planck. The combination $\mathrm{BAO}+\mathrm{CMB}$ represents the cosmological probes tied to high redshift.

The interplay between the shifts in the sound horizon and the distances acts to bias $w_{0}$ by a substantial $\Delta w_{0}=$ 0.29 , and mimic an evolution $w_{a}=-0.66$. More precise $\mathrm{BAO}$ measurements would lead to even greater relative bias. Note that in other models for EDE, such as the mocker model considered in [8], BAO can give even larger biases for the same amount of EDE.

Observations that are not calibrated off the high redshift universe do not suffer this miscalibration bias. Figure 3 shows the confidence region for luminosity distance measurements through Type Ia supernovae (SN), at $1 \%$ accuracy over the range $z=0-1.7$ as from the proposed SNAP mission, combined with a prior on the matter density mimicking what might be delivered by its gravitational weak lensing measurements of distance ratios. These measurements recover in an unbiased manner the true expansion history over this redshift range. That is, the luminosity distance predictions of the best fit $\left(w_{0}, w_{a}\right)=(-0.95,0.15)$ model agree with the true EDE cosmology to better than $0.02 \%$. (The expansion 


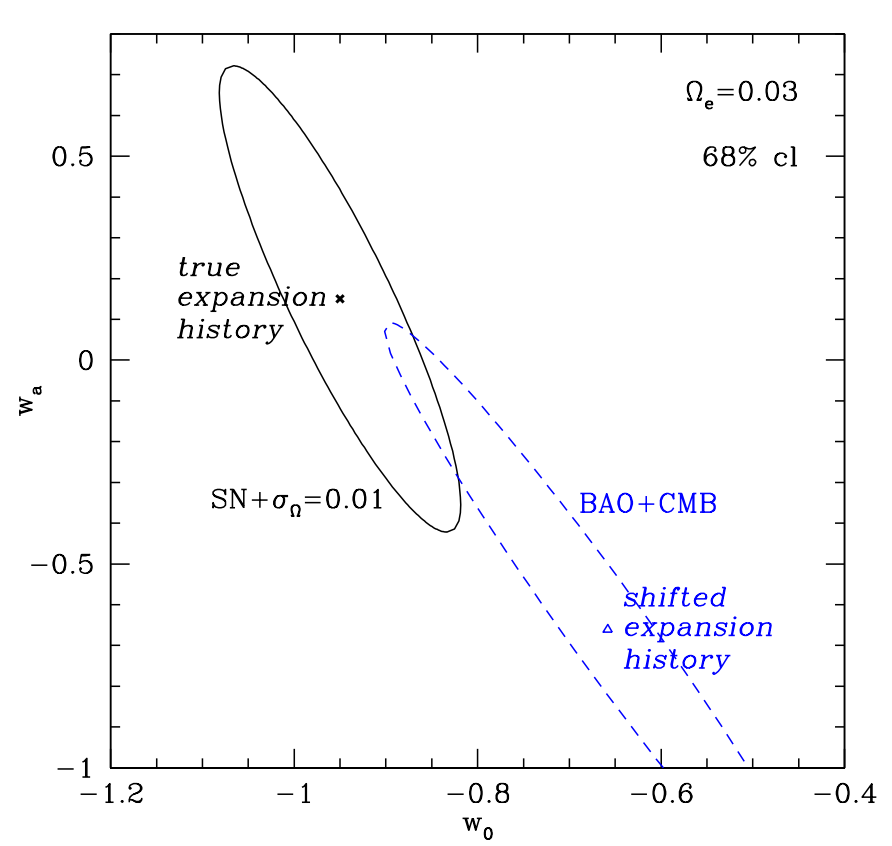

FIG. 3: Early dark energy density $\Omega_{e}$ can cause miscalibration of the standard ruler used by the baryon acoustic oscillation probe. This shifts the dark energy properties derived with $\mathrm{BAO}+\mathrm{CMB}$ data from their true values (black $\mathrm{x}$ ) to those marked by the blue triangle, with the dashed confidence contour. Distance probes such as supernovae that do not rely on the standard ruler recover the true expansion history.

history of an EDE model at $z \lesssim 2$ closely follows a standard dark energy model with the same $w_{0}$ and with an effective $w_{a} \approx 5 \Omega_{e}$.)

In order for $\mathrm{BAO}$ to recover the true cosmology, the technique must allow for the possibility of EDE; one must include a calibration parameter to standardize the ruler, i.e. a new parameter $s$ or $\delta s$. This is exactly analogous to the $\mathcal{M}$ parameter for $\mathrm{SN}$ that allows for the possibility that the low redshift calibration is not perfect, i.e. SN analysis does not assume they are known standard candles but fits for the absolute luminosity. When we include such a parameter in the fit, the bias goes away while the parameter estimation uncertainties increase of course.

The necessary presence of a standard ruler calibration parameter, call it $\mathcal{S}$, leads to an increase in the $w_{0}-w_{a}$ contour area, and equivalent decrease in the area "figure of merit" by a factor 2.3. (To decrease appreciably the area blowup would require prior constraint on $\mathcal{S}$ to better than $0.5 \%$.) Since we do not know a priori whether the high redshift universe is shifted by EDE, or by other physics mentioned in $₫ \underline{I I}$, neglecting $\mathcal{S}$ for BAO is as improper as neglecting $\mathcal{M}$ for supernova standard candle calibration. Without the need to fit for the low redshift calibration $\mathcal{M}$, SN would enjoy an improvement in "figure of merit" by a factor 1.9 , similar to the 2.3 that BAO has when neglecting the high redshift calibration $\mathcal{S}$. In summary, if calibration is ignored then BAO may deliver biased contours as in Fig. 3, while if calibration is implemented as described then the BAO contours are centered on the true cosmology but increase by $\sim 2.3$ in area.

\section{CONCLUSION}

Early dark energy has possible motivations both theoretically and observationally, but more than that we simply should not assume dark energy is a purely recent phenomenon. Such an assumption can significantly bias our understanding of the cosmological model even at low redshift. We have shown that recognizing early dark energy in the cosmic microwave background is not trivial, even if it significantly affects the size of the sound horizon.

A level of $\Omega_{e}>0.03$ would likely provide a recognizable signal in advanced CMB data. However, lesser amounts of early dark energy capable of shifting the universe by $\sim 1 \%$ are more difficult to identify robustly.

The sound horizon is a crucial quantity because it is employed as a standard ruler in the baryon acoustic oscillation technique for probing cosmology. If this standard ruler is miscalibrated due to unrecognized early dark energy, then bias will enter into interpretation of $\mathrm{BAO}$ scale measurements. The remedy is inclusion of a calibration parameter, just as exists for the supernova standard candle technique, which removes the bias but weakens the BAO constraints by a factor $\sim 2$.

Probing, rather than assuming the lack of, early dark energy offers an intriguing window on the new physics behind the accelerating universe. Structure formation as probed through the first stars, cluster abundances and the Sunyaev-Zel'dovich effect, and weak gravitational lensing may hold important clues to early dark energy, while precision measurements of the Hubble constant can provide additional leverage for CMB constraints. Understanding dark energy throughout the history of the universe is an exciting prospect.

\section{Acknowledgments}

EL thanks Christof Wetterich for hospitality at ITP Heidelberg during the course of this project, and Michael Doran and Martin White for useful conversations. This work has been supported in part by the Director, Office of Science, Department of Energy under grant DE-AC0205CH11231. GR acknowledges support by the Deutsche Forschungsgemeinschaft, grant TRR33 "The Dark Universe". 
[1] R. de Putter \& E.V. Linder, arXiv:0710.0373

[2] E.L. Wright, ApJ 664, 633 arXiv:astro-ph/0701584

[3] M. Kowalski et al., ApJ submitted

[4] M. Bartelmann, M. Doran, C. Wetterich, A\&A 454, 27 (2006) arXiv:astro-ph/0507257

[5] E.V. Linder, Astropart. Phys. 26, 16 (2006) arXiv:astro-ph/0603584

[6] D.J. Eisenstein \& M. White, Phys. Rev. D 70, 103523 (2004) arXiv:astro-ph/0407539

[7] M. Doran, S. Stern, E. Thommes, JCAP 0704, 015 (2007) [arXiv: astro-ph/0609075]

[8] E.V. Linder, J. Phys. A 40, 6697 (2007) arXiv:astro-ph/0612102

[9] I. Zlatev, L. Wang, P.J. Steinhardt, Phys. Rev. Lett. 82, 896 (1999) arXiv:astro-ph/9807002

[10] C. Wetterich, Nucl. Phys. B 302, 668 (1988)

[11] S. Dodelson, M. Kaplinghat, E. Stewart, Phys. Rev. Lett. 85, 5276 (2000) arXiv:astro-ph/0002360

[12] G. Barenboim \& J. Lykken, Phys. Lett. B 633, 453 (2006) arXiv:astro-ph/0504090
[13] L. Amendola \& S. Tsujikawa, Phys. Lett. B 660, 125 (2008) arXiv:0705.0396

[14] L. Amendola, Phys. Rev. D 69, 103524 (2004) arXiv:astro-ph/0311175

[15] M. Doran, G. Robbers, C. Wetterich, Phys. Rev. D 75, 023003 (2007) arXiv:astro-ph/0609814

[16] S. Sadeh, Y. Rephaeli, J. Silk, MNRAS 380, 637 (2007) arXiv:0706.1340

[17] M. Doran \& G. Robbers, JCAP 0606, 026 (2006) arXiv:astro-ph/0601544

[18] M. Doran, M. Lilley, J. Schwindt, C. Wetterich, Ap. J. 559, 501 (2001) arXiv:astro-ph/0012139

[19] W. Hu \& S. Dodelson, ARAA 40, 171 (2002) arXiv:astro-ph/0110414

[20] M. Doran, JCAP 0510, $011 \quad$ (2005) arXiv:astro-ph/0302138; http://www.cmbeasy.org

[21] M. Doran and C. M. Mueller, JCAP 0409, 003 (2004) arXiv:astro-ph/0311311.

[22] M.R. Nolta et al., arXiv:0803.0593 